ARTICLE

Received 20 Oct 2015 | Accepted 7 Apr 2016 | Published 18 May 2016

DOI: $10.1038 /$ ncomms11553

OPEN

\title{
Globally prevalent PfMDR1 mutations modulate Plasmodium falciparum susceptibility to artemisinin-based combination therapies
}

\author{
M. Isabel Veiga ${ }^{1,2, \star}$, Satish K. Dhingra ${ }^{1, \star}$, Philipp P. Henrich ${ }^{1}$, Judith Straimer ${ }^{1}$, Nina Gnädig ${ }^{1}$, \\ Anne-Catrin Uhlemann ${ }^{3}$, Rowena E. Martin ${ }^{4}$, Adele M. Lehane ${ }^{1,4}$ \& David A. Fidock ${ }^{1,3}$
}

Antimalarial chemotherapy, globally reliant on artemisinin-based combination therapies (ACTs), is threatened by the spread of drug resistance in Plasmodium falciparum parasites. Here we use zinc-finger nucleases to genetically modify the multidrug resistance-1 transporter PfMDR1 at amino acids 86 and 184, and demonstrate that the widely prevalent N86Y mutation augments resistance to the ACT partner drug amodiaquine and the former first-line agent chloroquine. In contrast, N86Y increases parasite susceptibility to the partner drugs lumefantrine and mefloquine, and the active artemisinin metabolite dihydroartemisinin. The PfMDR1 N86 plus Y184F isoform moderately reduces piperaquine potency in strains expressing an Asian/African variant of the chloroquine resistance transporter PfCRT. Mutations in both digestive vacuole-resident transporters are thought to differentially regulate $\mathrm{ACT}$ drug interactions with host haem, a product of parasite-mediated haemoglobin degradation. Global mapping of these mutations illustrates where the different ACTs could be selectively deployed to optimize treatment based on regional differences in PfMDR1 haplotypes.

\footnotetext{
${ }^{1}$ Department of Microbiology and Immunology, Columbia University Medical Center, Room 1502 HHSC, 701 West 168th Street, New York, New York 10032 USA. ${ }^{2}$ Life and Health Sciences Research Institute (ICVS), School of Health Sciences and ICVS/3B's-PT Government Associate Laboratory, University of Minho, Gualtar Campus, 4710-057 Braga, Portugal. ${ }^{3}$ Division of Infectious Diseases, Department of Medicine, Columbia University Medical Center, Box 82, 630 West 168th Street, New York, New York 10032, USA. ${ }^{4}$ Research School of Biology, Linnaeus Way, The Australian National University, Acton Australian Capital Territory 2601, Australia. ${ }^{\star}$ These authors contributed equally to this work. Correspondence and requests for materials should be addressed to D.A.F. (email: df2260@cumc.columbia.edu).
} 
$\mathrm{M}$ alaria in 2015 was responsible for an estimated 214 million cases and 438,000 deaths ${ }^{1}$. Fatal cases, resulting primarily from infection with the Apicomplexan parasite Plasmodium falciparum, are half those occurring 15 years ago, primarily as a result of the global adoption of highly effective artemisinin (ART)-based combination therapies (ACTs). These ACTs combine a fast acting but rapidly cleared ART derivative with a longer-lasting partner drug. The major partner drugs are lumefantrine (LMF, which combined with artemether (ATM) constitutes the most widely used ACT), mefloquine (MFQ, paired with artesunate (AS)), amodiaquine (ADQ, also paired with AS) and piperaquine (PPQ, combined with the active ART metabolite dihydroartemisinin (DHA)). These drugs share certain chemical features such as the 4-aminoquinoline ring present in $\mathrm{ADQ}$ and $\mathrm{PPQ}$ (as well as chloroquine, $\mathrm{CQ}$ ) or the arylaminoalcohol group present in LMF and MFQ (Fig. 1), and appear to interact with the parasite haem detoxification pathway ${ }^{2}$. These interactions might only be a part of complex modes of action, such as for MFQ (and possibly LMF) where activity against a cytosolic target (or targets) has been evoked ${ }^{3}$. In the case of ART derivatives, these are activated by the central $\mathrm{Fe}^{2+}$ atom present in haem on its release from haemoglobin ${ }^{4}$.

In a direct threat to malaria control programmes, ACT resistance is now emerging, led by $P$. falciparum resistance to the ART derivatives. This resistance, which manifests as relatively slow rates of parasite clearance following treatment, is now widespread in the Greater Mekong Sub-region ${ }^{5}$, raising concerns about its possible spread into the African continent where malaria exerts its heaviest toll. Epidemiological and molecular genetic studies have recently shown that ART resistance is primarily mediated by mutations in the propeller domain of the P. falciparum K13 kelch protein ${ }^{5-8}$. Reduced ART efficacy in turn places increased selective pressure on the ACT partner drugs, placing them at greater risk of failing. Indeed, $\mathrm{PPQ}$ resistance has now emerged in K13-mutant parasites, resulting in treatment failure with DHA + PPQ-the first-line drug in Cambodia and several neighbouring countries ${ }^{9-11}$. Despite an active antimalarial drug discovery and development pipeline, there is at present no drug ready to take the place of ACTs should they begin to fail globally ${ }^{12}$.

Delineating the role of the P. falciparum multidrug resistance-1 gene pfmdrl is of particular relevance due to its suspected involvement in parasite susceptibility to each of the ACT partner drugs mentioned above, as well as its association with altered susceptibilities of trophozoite-stage parasites to ART derivatives ${ }^{13-15}$. These findings, acquired in molecular

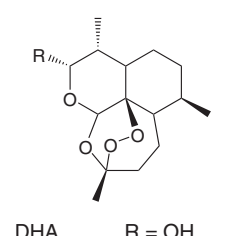

$\begin{array}{ll}\mathrm{DHA} & \mathrm{R}=\mathrm{OH} \\ \text { Artemether } & \mathrm{R}=\mathrm{OCH}\end{array}$

Artesunate $\mathrm{R}=\mathrm{OCOC}_{2} \mathrm{H}_{4} \mathrm{COOH} \quad$ Lumefantrine

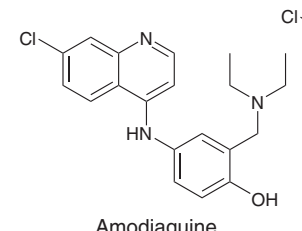

Amodiaquine

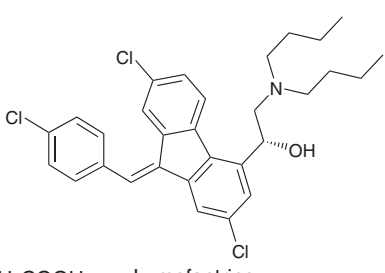

Cl

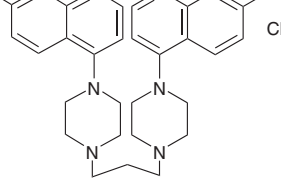

Piperaquine

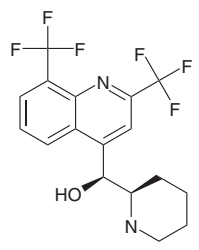

Mefloquine
Figure 1 | Chemical structures of antimalarials used in ACTs. Clinically used artemisinin derivatives are shown on the top left. DHA, dihydroartemisinin. epidemiology studies, implicate mutant PfMDR1 in multidrug resistance phenotypes. The interpretation of these earlier studies, which largely relied on typing single nucleotide polymorphisms (SNPs), is tempered by the lack of complete PfMDR1 haplotypes. Studies of isogenic parasites engineered to differ only at their $p f m d r 1$ locus have the benefit of reducing the genetic complexity and attributing changes in drug susceptibility to the introduced sequence changes. Such studies have become more technically feasible since the advent of genome editing in P. falciparum, which significantly increases the speed and precision with which SNPs can be exchanged at a gene of interest ${ }^{16}$.

PfMDR1 is an ATP-binding cassette transporter and a homologue of the human multidrug-resistance-conferring P-glycoprotein. It is 1,419 amino acids in length (for the 3D7 isoform) and contains 12 putative transmembrane domains. PfMDR1 and the pleiotropic drug resistance transporter PfCRT both reside on the membrane of the parasite's digestive vacuole (DV), where they are thought to regulate the flux of solutes across this membrane ${ }^{17}$. Their functional relatedness is evidenced by the linkage disequilibrium seen between certain combinations of pfmdr1 and pfcrt alleles ${ }^{18,19}$. This might serve to maximize drug resistance phenotypes and/or reflect compensatory mutations that reduce any negative impact of mutations in one transporter on DV physiology or parasite growth. In a genetic cross between clones of South American (HB3, CQ sensitive) and Asian (Dd2, CQ resistant) origin, pfcrt was identified as the primary determinant of CQ resistance, with the K76T mutation being ubiquitous to the CQ-resistant progeny ${ }^{20}$. A separate cross between CQ-resistant clones from South America (7G8) and Africa (GB4; having a higher degree of CQ resistance than 7G8) revealed that the South American pfcrt and pfmdr1 alleles combine to confer high-level resistance to monodesethyl-ADQ (md-ADQ), the active ADQ metabolite ${ }^{19}$.

Studies of $p f m d r 1$ have identified five globally prevalent amino acid mutations. The amino-terminal mutations (N86Y and Y184F) are more common to Asian and African parasites, whereas the three carboxy-terminal mutations (S1034C, N1042D and D1246Y) are found more often in South American isolates (D1246Y is nonetheless present in $\sim 3 \%$ of the 1,502 African genomes recently sequenced by the MalariaGEN consortium; see below). The ability of PfMDR1 variants to influence antimalarial drug potency is supported by heterologous expression systems that provide evidence of drug transport by certain PfMDR1 isoforms $\mathrm{s}^{21,22}$.

Earlier transfection studies have delineated the role of the C-terminal PfMDR1 mutations in modulating P. falciparum response to antimalarial drugs including MFQ, ART, CQ and quinine $(\mathrm{QN})^{23,24}$. Attempts to modify the N-terminal mutations were unsuccessful, presumably because the former single-site, cross-over-based strategies necessitated changes to the pfmdr1 regulatory elements that proved unsuitable for parasite growth ${ }^{24}$. That restriction has been negated with the development of zincfinger nucleases (ZFNs), which permit precise gene editing by triggering a specific double-stranded break adjacent to the targeted SNP. Homology-directed recombination can then be leveraged to repair the DNA lesion, without requiring the modification of any gene regulatory elements or the permanent integration of a selectable marker ${ }^{25}$. This approach has been successfully used to define the role of the resistance mediators $p f c r t, k 13$ and pi4k $k^{7,26-28}$. Here we report its successful use to engineer pfmdr1 N86Y and Y184F mutations in parasite strains that express the two major CQ resistance-conferring PfCRT variants. We also use publicly available data from 2,512 P. falciparum genomes to explore the distribution of PfMDR1 haplotypes at positions 86 and 184 in endemic regions. Our results show that the N86Y mutation contributes to 
resistance to $\mathrm{CQ}$ and $\mathrm{ADQ}$, while sensitizing parasites to $\mathrm{LMF}$, MFQ and DHA. In contrast, the Y184F mutation has a limited impact. When combined with the genome analyses, these findings help inform the selection of optimal treatment regimens based on an assessment of local drug selective pressures and the geographic distribution of PfMDR1 haplotypes.

\section{Results}

Geographical distribution of PfMDR1 haplotypes. Recent advances in whole-genome sequencing and genome analysis, applied to thousands of $P$. falciparum genomes by the MalariaGEN consortium ${ }^{8,29}$, permit a detailed investigation of PfMDR1 haplotypes across malaria-endemic regions of Southeast Asia and Africa (large-scale genome data from South American and Western Pacific strains are not yet available). By analysing 2,512 parasite genomes made available by this consortium (www.malariagen.net/pf3k), we determined the frequencies of the four PfMDR1 haplotypes differing at residues 86 and 184 across multiple countries (Fig. 2 and Supplementary Table 1). We used the N86/Y184 (NY) haplotype of the drug-sensitive reference strain 3D7 as the wild-type comparator. Results show three of the four haplotypes at positions 86 and 184 being relatively common across West Africa, with only N86Y/Y184 PfMDR1 (YY) being relatively rare. All four were observed in the Congo in Central Africa, while Malawi in East Africa essentially lacked the N86Y mutation and harboured only N86/Y184F (NF) and NY. In Southeast Asia the N86Y mutation was also strikingly absent (although it remained to the west in Bangladesh) and the Y184F mutation varied in prevalence from $>50 \%$ in Cambodia to $<4 \%$ in Laos. Southeast Asian isolates quite often also harboured multiple copies of pfmdr1 (155/1,010 genomes), more so in parasites with the NY haplotype and never with the N86Y mutation (Fig. 2 and Supplementary Table 1). This was most apparent in Thailand, where nearly $40 \%$ of all genomes had amplified $p f m d r 1$, with as many as five tandem copies. Amplified pfmdr1 was exceedingly rare in Africa (observed in 12/1,502 genomes), potentially because of a fitness cost that is not conducive to its maintenance in African high-endemicity settings ${ }^{30}$. Parallel assessment of pfcrt codon 76 revealed that 98\% of all sampled parasites in Southeast Asia carried the K76T mutation (Table 1 and Supplementary Table 1). Surveys of African parasites revealed far greater heterogeneity. Parasites from Malawi were exclusively K76, consistent with strict removal of CQ pressure ${ }^{31}$, whereas other countries such as the Congo maintained the K76T CQ resistance marker in $71 \%$ of the sampled isolates.

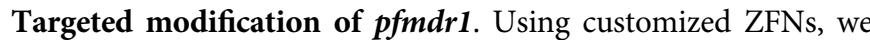
generated two sets of pfmdrl-edited parasites at codons 86 and 184 (Table 2). These sets represented the two major geographic variants of mutant PfCRT, namely SVMNT and CVIET (spanning codons 72-76). ZFNs were engineered to bind neighbouring sites on opposite strands of $p f m d r 1$, producing a double-stranded break $42 \mathrm{bp}$ downstream of the start codon. As previously documented, double-stranded break repairs proceed via homology-directed recombination in P. falciparum, which lacks the alternative non-homologous end-joining repair pathway ${ }^{25}$. Our homology-driven repair template consisted of a 2.4-kb pfmdr1 fragment that encompassed codons 86 and 184. To prevent ZFNs from cleaving the plasmid or the edited locus, three silent (non-synonymous) mutations were engineered into the donor sequence at the ZFN-binding site.

P. falciparum-infected red blood cells (RBCs), predominantly ring stages, were electroporated with the pmdr1 ZFN plasmids that also express human dihydrofolate reductase (hDHFR).
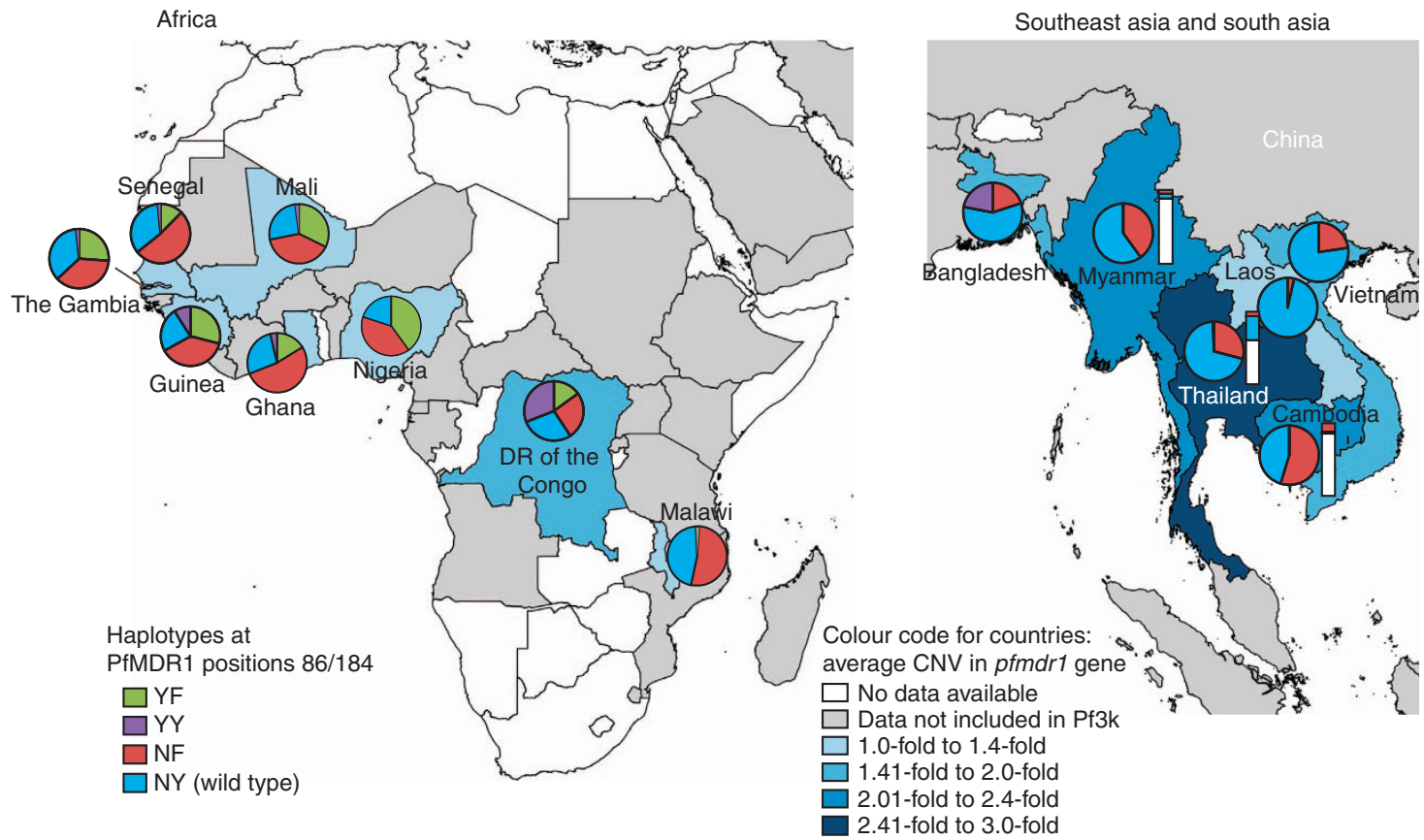

Figure 2 | Geographical distribution of PfMDR1 haplotypes at residues 86 and $\mathbf{1 8 4}$ and pfmdr1 copy number variations. Data were compiled using the Sanger Pf3k data set (www.malariagen.net/apps/pf3k/release_3/index.html) ${ }^{8,29}$ and comprised 2,512 genomes. Pie charts represent proportions of PfMDR1 haplotypes (numerical data per country in Supplementary Table 1). Country colourings show average pfmdr1 amplification compared with the 3D7 reference genome with single-copy pfmdr1. Vertical bars in Southeast Asia show the proportions of haplotypes represented among pfmdr1 copy number variants. Vertical bars colouring: White: no pfmdr1 amplification; blue: pfmdr1 amplification plus NY haplotype; red: pfmdr1 amplification plus NF haplotype. Countries where PfMDR1 individual polymorphism data are available via WWARN (www.wwarn.org/tracking-resistance/molecular-surveyor-pfmdr1-pfcrt) are indicated in grey (data not evaluated herein due to the lack of combined N86 and Y184 status in the full data set). 
Table 1 | Prevalence of correlated PfCRT-PfMDR1 haplotypes.

\begin{tabular}{|c|c|c|c|c|c|c|c|c|}
\hline \multicolumn{5}{|c|}{ PfCRT haplotype } & \multicolumn{2}{|c|}{ PfMDR1 haplotype } & \multirow{2}{*}{$\begin{array}{c}\text { African correlation } \\
\text { Prevalence (isolates) }\end{array}$} & \multirow{2}{*}{$\begin{array}{c}\text { Southeast Asian correlation } \\
\text { Prevalence (isolates) } \\
\end{array}$} \\
\hline 72 & 73 & 74 & 75 & 76 & 86 & 184 & & \\
\hline C & $\mathrm{V}$ & $M$ & $\mathrm{~N}$ & $\mathrm{~K}$ & $Y$ & $\mathrm{~F}$ & $8.4 \%(126)$ & ND \\
\hline C & V & M & $\mathrm{N}$ & K & Y & Y & $1.8 \%(27)$ & ND \\
\hline C & $\mathrm{V}$ & M & $\mathrm{N}$ & K & $\mathrm{N}$ & Y & $20.9 \%(314)$ & $1.7 \%(16)$ \\
\hline C & $\mathrm{V}$ & 1 & $E$ & $\mathrm{~T}$ & Y & $\mathrm{F}$ & $8.1 \%(121)$ & ND \\
\hline C & $\mathrm{V}$ & 1 & $E$ & $\mathrm{~T}$ & Y & Y & $3.1 \%(46)$ & $0.1 \%(1)$ \\
\hline C & $\mathrm{V}$ & 1 & D & $\mathrm{T}$ & $Y$ & $\mathrm{~F}$ & ND & ND \\
\hline C & $\mathrm{V}$ & 1 & D & $\mathrm{T}$ & $\mathrm{Y}$ & Y & ND & ND \\
\hline C & $\mathrm{V}$ & 1 & D & $\mathrm{T}$ & $\mathrm{N}$ & $\mathrm{F}$ & ND & $2.1 \%(20)$ \\
\hline C & $\mathrm{V}$ & 1 & D & $\mathrm{T}$ & $\mathrm{N}$ & Y & ND & $15.1 \%(145)$ \\
\hline & & & & & & Total & $97.9 \%(1,469)$ & $99.6 \%(956)$ \\
\hline
\end{tabular}

ND, not detected.

This table illustrates the major PfCRT and PfMDR1 haplotypes in Asian and African isolates whose genome sequences have been deposited in the Pf3k database (www.malariagen.net/pf3k). The remaining minor fractions consist of non-canonical PfCRT haplotpyes.

Table 2 | Haplotypes of PfMDR1-modified and control clones.

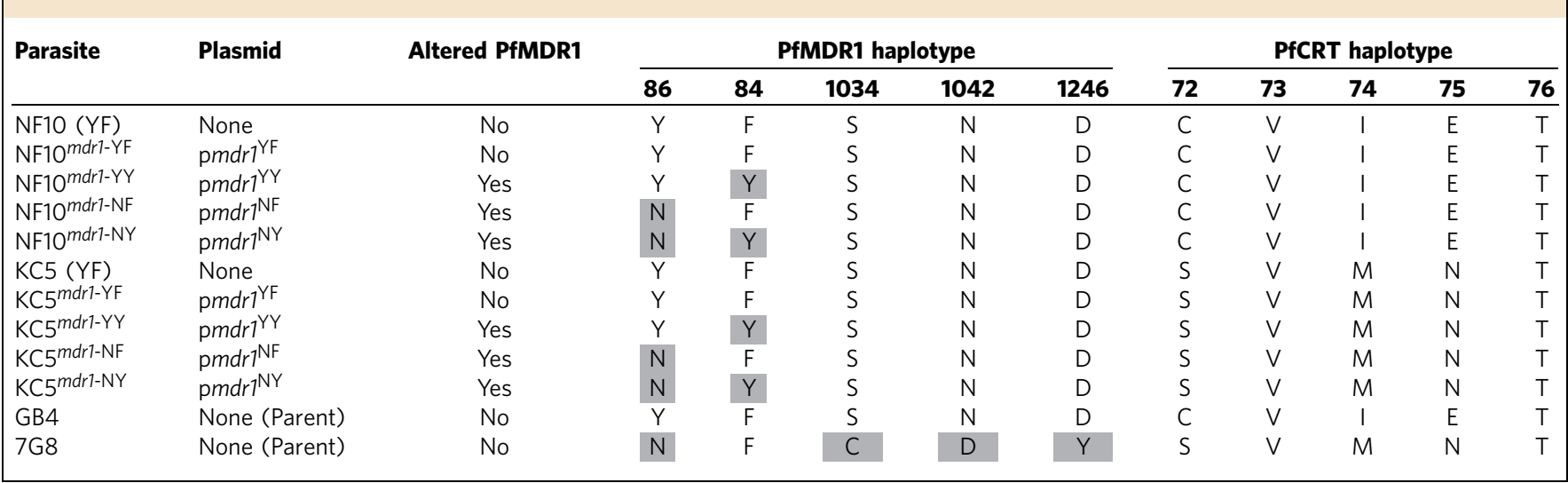

This selectable marker mediates resistance to WR99210, which was applied for 4-6 days, to select for transformed parasites. PCR screening was used to identify edited parasites, which were cloned by limiting dilution. Figure 3 illustrates the ZFN strategy and shows representative electropherograms of $p f m d r 1$ editing events.

Editing of pfmdr1 was performed with the P. falciparum NF10 and $\mathrm{KC} 5$ clones. These are progeny of the genetic cross between $7 \mathrm{G} 8$ (Brazil) and GB4 (Ghana) parasites (Table 2) ${ }^{32}$. Both progeny lines inherited a single copy of the GB4 pfmdr1 allele, which encodes the N86Y/Y184F haplotype (YF). NF10 inherited the CVIET PfCRT haplotype that is predominant in Asia and Africa, whereas KC5 harbours the SVMNT haplotype that is highly prevalent in South America and the Western Pacific. These progeny differ in their level of CQ resistance and their response to $\mathrm{QN}$ and $\mathrm{ADQ}^{19}$ (see below). Parasites were transfected with four different $\mathrm{p} m d r 1$ plasmids to generate the four different haplotype combinations, namely YF, YY, NF and NY, in both pfcrt genetic backgrounds. The resulting lines are denoted NF10 mdrl-YF (recombinant control), NF10 $0^{m}$ rr $1-\mathrm{YY}, \mathrm{NF} 10^{m d r 1-\mathrm{NF}}, \mathrm{NF} 10^{m d r 1-\mathrm{NY}}$, $\mathrm{KC} 5^{m d r 1-\mathrm{YF}}$ (recombinant control), KC5 ${ }^{m d r 1-\mathrm{YY}}, \mathrm{KC}^{\text {mdrl }-\mathrm{NF}}$ and KC5 ${ }^{\text {mdr1-NY }}$ (Table 2). Quantitative PCR analysis revealed that all parasites continued to express a single copy of $p f m d r l$, with no change introduced during gene editing (Supplementary Table 2).

In vitro drug susceptibility assays were performed on the different $p f m d r 1$ recombinant lines. Assays were performed with two independent clones for each line, with the exception of the recombinant control lines (NF10 ${ }^{\text {mdr1-YF-1 }}$ and KC $5^{\text {mdr1-YF-1 }}$ ) and the $\mathrm{KC} 5^{\text {mdr- } \mathrm{YY}}$ line for which only single clones were recovered. Drug susceptibility assays used a range of ten compound concentrations diluted twofold and were performed on five to ten separate occasions in duplicate. Numbers of surviving parasites were quantified using flow cytometry. $\mathrm{IC}_{50}$ and $\mathrm{IC}_{90}$ values were determined for LMF, MFQ, DHA, PPQ, CQ, monodesethyl-CQ (md-CQ; the major in vivo metabolite of $\mathrm{CQ})$, md-ADQ and QN.

Drug assays with gene-edited control parasites carrying only silent ZFN binding-site mutations showed no change in $\mathrm{IC}_{50}$ values relative to the parental lines (see NF10 mdrr-YF-1 versus NF10 and KC5 ${ }^{\text {mdrl-YF-1 }}$ versus KC5; Figs 4 and 5). Mean \pm s.e.m. $\mathrm{IC}_{50}$ and $\mathrm{IC}_{90}$ values, numbers of independent assays performed and Mann-Whitney tests for statistical significance are listed in Supplementary Tables 3-6. Control assays with the antimalarial drug atovaquone, which targets the mitochondrial bc1 complex ${ }^{33}$, showed no changes in $\mathrm{IC}_{50}$ values between any of the $p f m d r 1$ recombinant lines and their parental controls (Supplementary Fig. 1).

PfMDR1 N86Y increases susceptibility to arylaminoalcohols and DHA. The most striking phenotype observed with the replacement of N86Y with the wild-type N86 residue was a significant increase in the $\mathrm{IC}_{50}$ and $\mathrm{IC}_{90}$ values for $\mathrm{LMF}, \mathrm{MFQ}$ and DHA (Fig. 4 and Supplementary Tables 3-6). The change to N86 resulted in a three- to fourfold increase in the $\mathrm{IC}_{50}$ values for LMF 


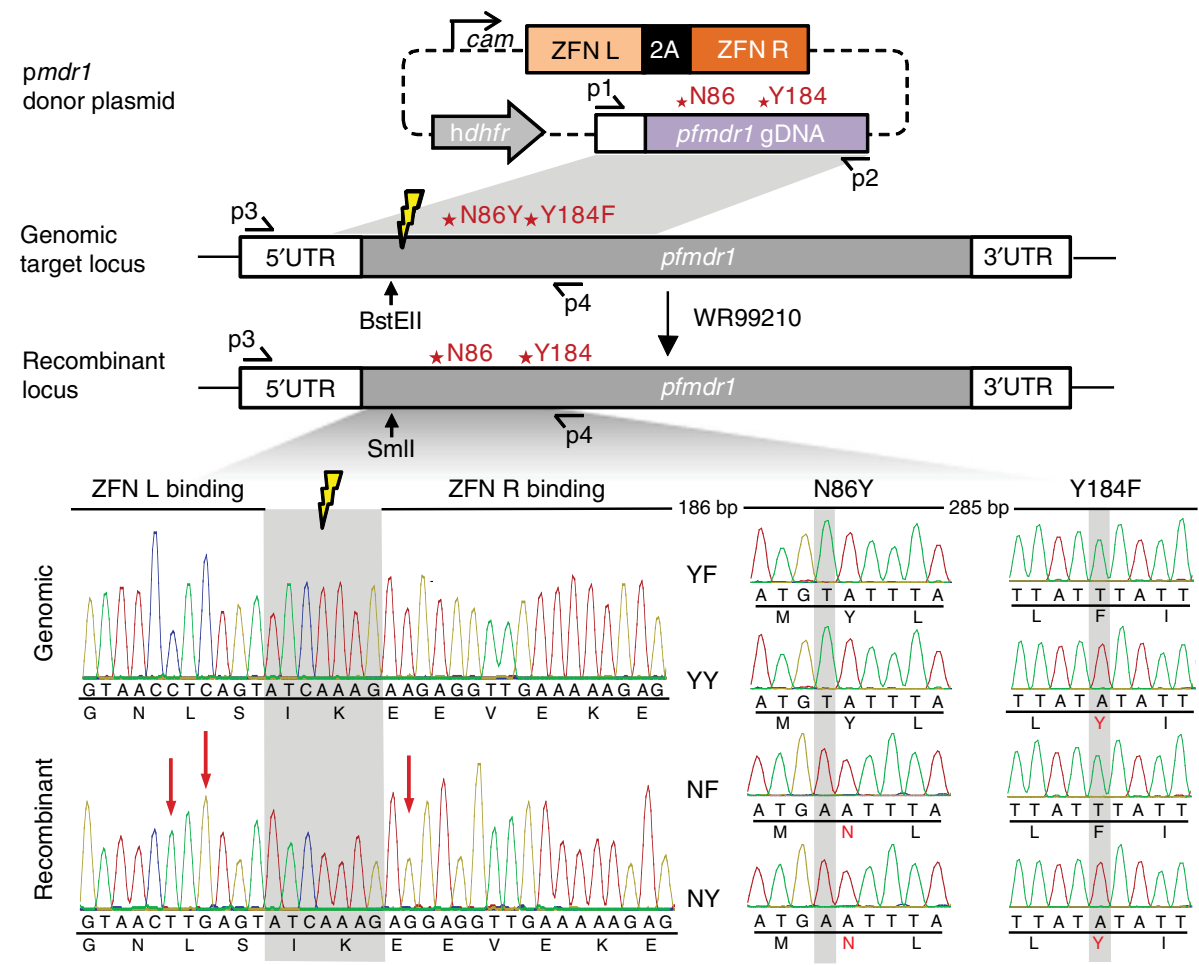

Figure 3 | Schematic of the ZFN-based pfmdr1 editing strategy. The pmdr1 plasmid expresses the 2A-linked pfmdr1-specific ZFN pair from the calmodulin promoter and the human dhfr selectable marker. These ZFNs create a double-stranded break that can be repaired using the plasmid-borne homologous donor sequence that extends $0.6 \mathrm{~kb}$ upstream and $1.8 \mathrm{~kb}$ downstream of the target site (yellow thunderbolt). The four pmdr 1 transfection plasmids carry three silent mutations at the binding sites to prevent ZFNs cleaving the plasmids or the edited sequence. These plasmids encode the four haplotypes at residues 86 and 184. Shown is the example of transfecting a N86/Y184 parasite with the pmdr $7^{\mathrm{NY}}$ plasmid (Table 2). Chromatograms show sequence analysis of genomic and recombinant DNA samples. Mutations at the ZFN binding site (red arrows) and the N86Y and Y184F change of codons are indicated.

and MFQ in both the NF10 and KC5 backgrounds $(P<0.001$, Mann-Whitney $U$-test).

In the case of DHA, the change to N86 resulted in $\sim 1.5$-fold increased $\mathrm{IC}_{50}$ values in both backgrounds $(P<0.05)$. NF10 recombinant and parental lines were also subjected to in vitro survival assays, whereby highly synchronized early ring stages ( $0-3 \mathrm{~h}$ post-invasion) or trophozoites (18-21 $\mathrm{h}$ post-invasion) were exposed to $700 \mathrm{nM}$ DHA for $6 \mathrm{~h}$, followed by washing to remove drug and assessment of parasite growth $66 \mathrm{~h}$ later ${ }^{34}$. Results from three independent $\mathrm{RSA}_{0-3 \mathrm{~h}}$ and $\mathrm{TSA}_{18-21 \mathrm{~h}}$ assays found no significant difference between the various PfMDR1 haplotypes (Supplementary Fig. 2). As a control, we included the Cam3.II ${ }^{\mathrm{R} 539 \mathrm{~T}}$ line, which expresses the K13 R539T mutation that confers in vitro ring-stage resistance to $\mathrm{DHA}^{7}$. These results provide evidence that the differences observed in the $72 \mathrm{~h}$ in vitro inhibition assays did not translate into survival benefits on short-term exposure to a concentration of DHA.

PfMDR1 N86Y decreases susceptibility to CQ and md-ADQ. Our assays revealed that the parental NF10 line was less sensitive to $C Q$, md-CQ and QN but was more sensitive to md-ADQ when compared with the parental KC5 line. This is consistent with earlier studies of the $7 \mathrm{G} 8 \times \mathrm{GB} 4$ cross $^{19}$ that attributed these changes to the combined effects of PfCRT and PfMDR1. In the case of the NF10 and KC5 progeny that harbour the same PfMDR1 YF haplotype, these differences in drug response can be attributed primarily to mutations in PfCRT (CVIET and SVMNT, respectively). The replacement of N86Y with the wildtype N86 residue resulted in increased susceptibility to CQ, md$\mathrm{CQ}$ and md-ADQ with the greatest difference being observed in the NF10 recombinant parasites (Fig. 4).
For QN and PPQ, increased susceptibility was also observed on replacement of the N86Y to N86 in the NF10 line. However, this difference reached significance in only one of the NF and NY recombinant clones (NF-2 and NY-2) for $\mathrm{QN} \quad(P<0.05$, Mann-Whitney $U$-test; Fig. 5) and only in the NY recombinant line for PPQ $(P<0.01$; Fig. 4). We also investigated whether the PfMDR1 N86Y and Y184F mutations affected the accumulation of radiolabelled CQ, QN or ADQ in recombinant NF10 parasites. These assays revealed no statistically significant effect of the $\mathrm{N}$-terminal PfMDR1 mutations on the accumulation of these drugs by trophozoite-infected RBCs (Supplementary Fig. 3).

PfMDR1 Y184F has a minor impact on drug response. Compared with the N86Y mutation, the Y184F mutation in PfMDR1 appears to have a weaker association with antimalarial effectiveness in vivo ${ }^{13,35}$. Consistent with these observations, we report that differences in the antimalarial $\mathrm{IC}_{50}$ values between parasite lines expressing the PfMDR1 184 wild-type or mutant residue were dependent on the status of residue 86 , the class of drug and the pfcrt genetic background (Figs 4 and 5, and Supplementary Tables 3 and 4). In lines expressing N86Y, the presence of $\mathrm{Y}$ or $\mathrm{F}$ at position 184 had no detectable influence on parasite susceptibility to the drugs tested $(P>0.1$, Mann-Whitney $U$-test). In parasites with N86, isogenic Y184F and Y184 lines were generally very similar in their $\mathrm{IC}_{50}$ values for most drugs, with some exceptions presented below.

In the KC5 background, clones expressing the PfMDR1 $\mathrm{NF}$ haplotype presented slightly reduced $\mathrm{IC}_{50}$ values for LMF and MFQ when compared with the NY clones (Fig. 4 and Supplementary Table 4). In the NF10 background, the PfMDR1 $\mathrm{NF}$ clones exhibited higher $\mathrm{IC}_{50}$ values relative to the $\mathrm{NY}$ clones 

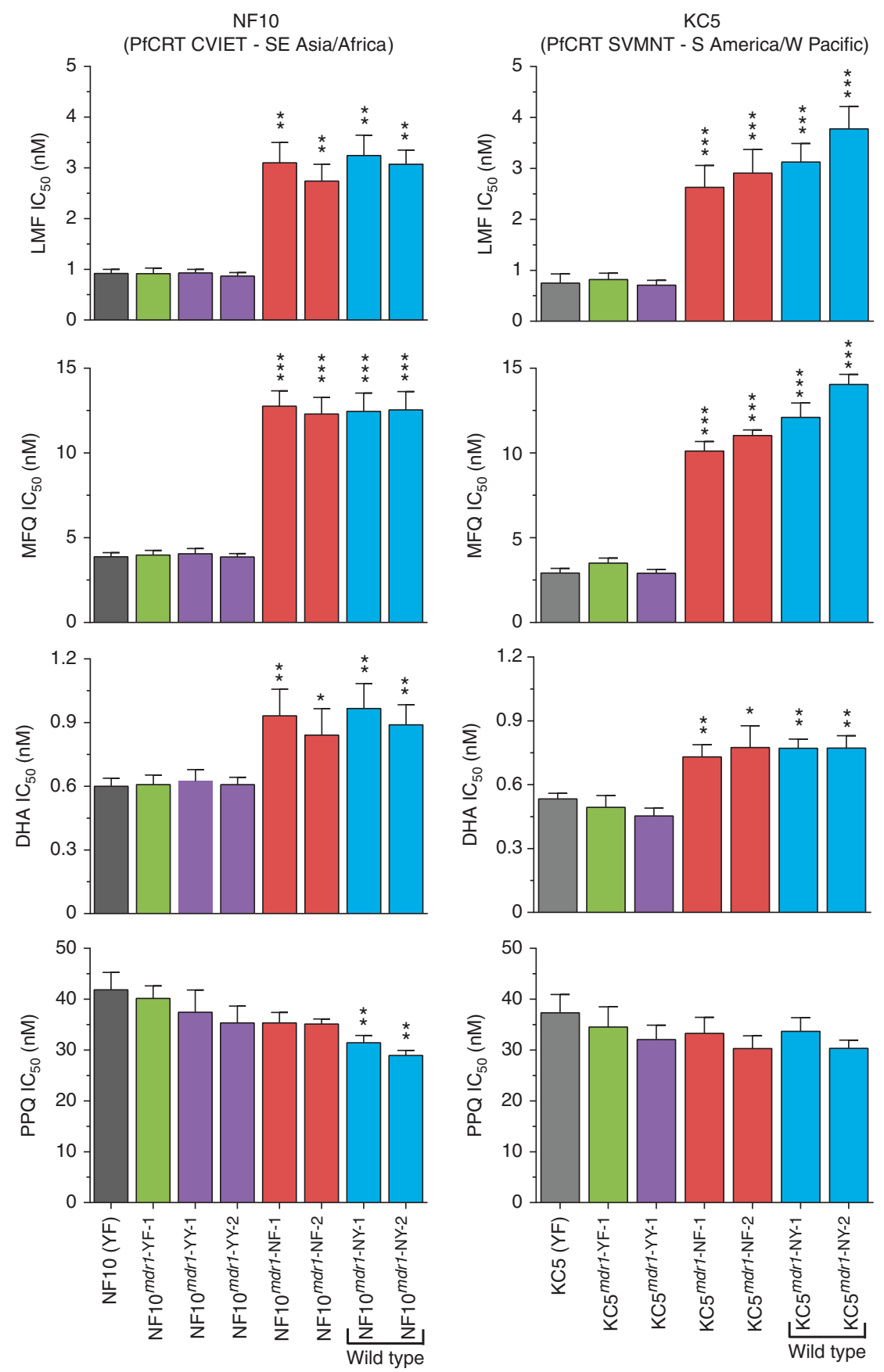

Figure 4 | In vitro IC I $_{\mathbf{5 0}}$ response of pfmdr1-modified and parental lines. $I C_{50}$ values $(n M)$ were determined by incubating parasites for $72 \mathrm{~h}$ across a range of drug concentrations. Parasite growth was determined by measuring parasitaemia using flow cytometry with cells stained with Mito Tracker Deep Red and SYBR Green. Mean \pm s.e.m. IC $C_{50}$ values are presented for lumefantrine (LMF), mefloquine (MFQ), dihydroartemisinin (DHA) and piperaquine (PPQ). Five to ten assays were performed for each drug (Supplementary Tables 3 and 4). Statistical evaluations comparing mutant pfmdr1-modified lines against recombinant control lines of the same genetic backgrounds (NF10 $0^{m d r-Y F-1}$ and KC $5^{\text {mdr1-YF-1 }}$ for NF10 and KC5 lines, respectively) were performed using two-tailed Mann-Whitney $U$-tests. ${ }^{\star} P<0.05,{ }^{\star \star} P<0.01$ and ${ }^{\star \star \star} P<0.001$.

for $\mathrm{CQ}$ and $\mathrm{PPQ}$, with significance achieved in one of the recombinant clones tested (Supplementary Table 3). As discussed below, this possible decrease in PPQ susceptibility in parasites expressing the PfMDR1 NF haplotype in the CVIET PfCRT background is consistent with the high prevalence of NF parasites in Cambodia, where PPQ is widely used.

In absolute numbers, our data revealed changes in susceptibility that remained within commonly employed cutoffs of resistance for $\mathrm{CQ}^{36}\left(\mathrm{IC}_{50}\right.$ values above $80 \mathrm{nM}$ ) or sensitivity for $\mathrm{MFQ}^{37}\left(\mathrm{IC}_{50}\right.$ values below $\left.15 \mathrm{nM}\right)$. Nevertheless, drug responses in our genome-edited parasites presented a notable fold change (up to fourfold for some of the drugs tested). Although these relative changes might not suffice to cause clinical failure, they may contribute to clinical outcome, in conjunction with other infection variables.

PfMDR1 F1226Y shows no impact on in vitro drug response. Recent studies have identified nearly $50 \%$ prevalence of the PfMDR1 F1226Y mutation in Thailand and Cambodia, and 

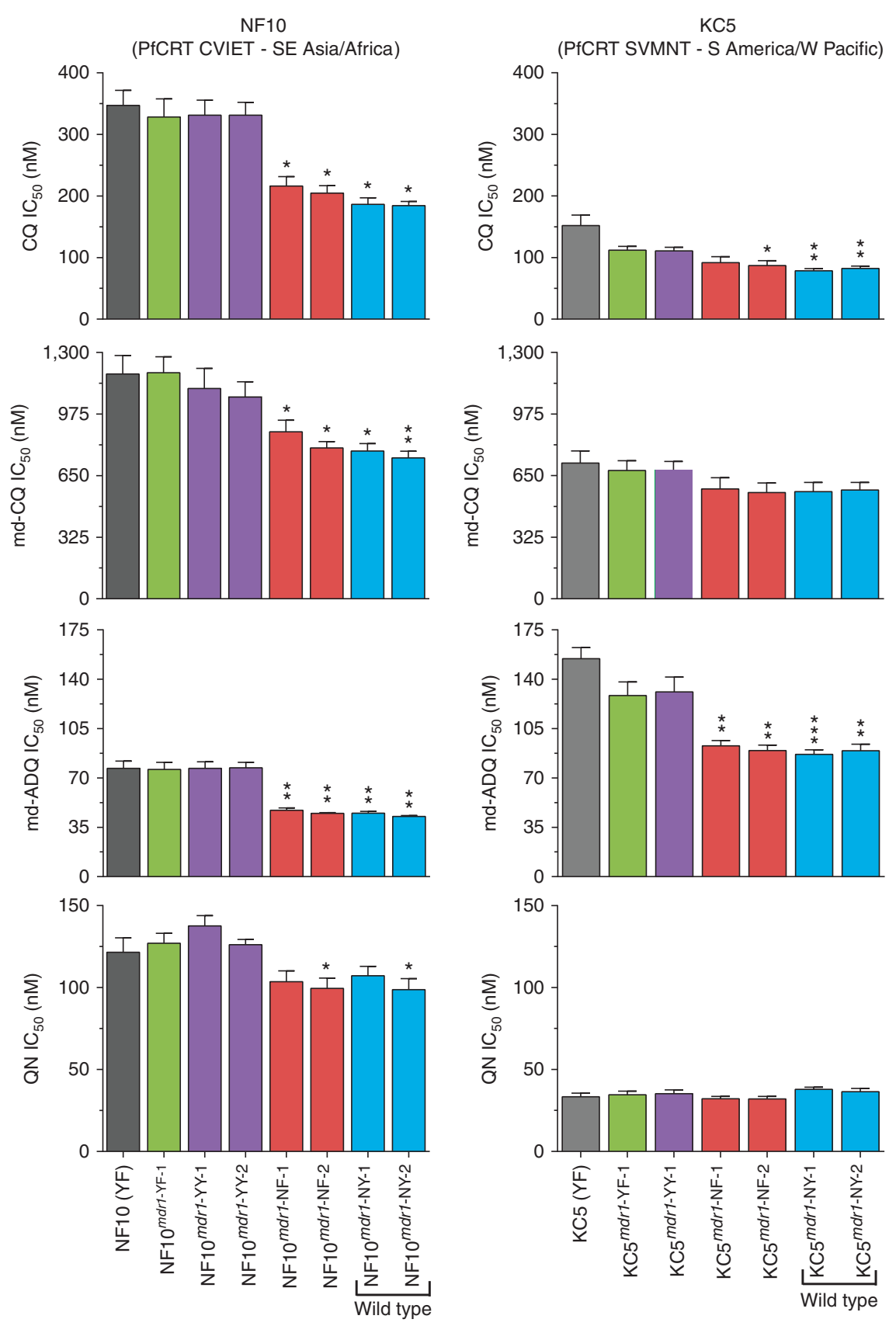

Figure 5 | In vitro $I_{\mathbf{5 0}}$ response of pfmdr1-modified and parental lines. $I C_{50}$ values $(n M)$ are presented for $C Q, m d-C Q$, $m d-A D Q$ and $Q N$. Five to ten assays were performed for each drug (Supplementary Tables 3 and 4). Statistical evaluations comparing mutant pfmdr1-modified lines against recombinant control lines of the same genetic backgrounds were performed using two-tailed Mann-Whitney $U$-tests, as described for Fig. 4 . ${ }^{\star} P<0.05$, ${ }^{\star \star} P<0.01$ and $\star \star \star P<0.001$.

suggested its contribution to altered antimalarial drug susceptibilities $^{38,39}$. To examine the role of this mutation in vitro, we employed a single cross-over-based allelic exchange strategy to introduce the PfMDR1 F1226Y mutation into the CQ-resistant K1 strain (obtained from Thailand in the early 1980s), which harbours a single copy of $p f m d r 1$ with YYSNFD at positions 86, 184, 1034, 1042, 1226 and 1246 (Supplementary Fig. 4). Phenotypic assessment of the parental strain and the F1226Y recombinant lines revealed no difference in the $\mathrm{IC}_{50}$ values for any of the drugs tested (LMF, MFQ, ART, AS, PPQ, CQ, md-ADQ and QN; Supplementary Table 7). Further studies will be required to assess whether this mutation might have an impact on drug susceptibilities in other Asian parasites, especially given that these no longer harbour the N86Y mutation and are often pfmdr1 multicopy (Fig. 2).

\section{Discussion}

Our study of genetically engineered $P$. falciparum lines with isogenic controls provides direct evidence that the PfMDR1 mutation N86Y modulates parasite susceptibility to a wide range of first-line antimalarials. We demonstrate that the N86Y mutation increases parasite susceptibility to LMF, MFQ and DHA, and conversely augments resistance to md-ADQ and CQ. The PfMDR1 Y184F mutation had a notably lesser impact in our isogenic lines, with evidence of decreasing $P$. falciparum 
susceptibility to PPQ when paired with the N86 residue. These data, which resolve long-standing questions about the role of PfMDR1, provide a foundation for improved molecular surveillance of antimalarial drug resistance and help inform region-specific decisions on which ACTs to employ.

The most striking effect of N86Y was on susceptibility to LMF and MFQ, for which parasites engineered to express wild-type N86 showed three- to fourfold higher drug $\mathrm{IC}_{50}$ values compared with the mutant N86Y residue. These data confirm genotyping and drug association studies performed with African and Asian isolates $^{40,41}$, and are consistent with reports that the N86 allele predominates in recurrent infections following treatment with $\mathrm{ATM}+\mathrm{LMF}^{13,42}$. Indeed, a recent meta-analysis of 31 clinical trials revealed that patients infected with N86 parasites had a fivefold greater risk of parasite recrudescence following ATM + LMF treatment compared with those infected with N86Y parasites ${ }^{15}$. Those data illustrate the utility of the in vitro data generated herein in identifying susceptibility shifts that correlate with changes in clinical outcomes.

The N86Y mutation was also observed to alter the parasite response to DHA, with $\sim 1.5$-fold decreased $\mathrm{IC}_{50}$ values compared with isogenic N86 parasites. Previous in vitro studies on laboratory strains and clinical isolates have also noted crossresistance between MFQ, LMF and ART derivatives ${ }^{23,24,35}$. This differs from the ART resistance mediated by mutations in K13, which manifests as reduced ring-stage susceptibility to $\mathrm{DHA}^{6,7}$. The $\mathrm{IC}_{50}$ shifts observed here are likely to reflect a role for $p f m d r 1$ in reducing parasite susceptibility at the trophozoite stage when haemoglobin degradation is the most active, producing high intracellular concentrations of the ART-activating ferrous iron that resides within haem ${ }^{43}$.

In contrast to our finding that the N86Y mutation increases parasite susceptibility to LMF, MFQ and DHA, we observed that this mutation decreases parasite susceptibility to CQ, md-CQ and md-ADQ in the NF10 background. CQ resistance has earlier been proposed to have some degree of association with the N86Y mutation, both in South American and Asian/African backgrounds ${ }^{44}$. Previous studies with patient isolates have also linked the N86Y mutation to reduced parasite susceptibility to $\mathrm{ADQ}^{45,46}$. Our data reveal that the effect of the N86Y mutation on parasite response to CQ and md-CQ is significant in parasites expressing the CVIET PfCRT variant. This effect, however, is modest in parasites expressing the SVMNT PfCRT variant. The latter observation is probably due, at least in part, to differences in the kinetics of PfCRT-mediated CQ and md-CQ transport between the high-capacity CVIET transporters and the low-capacity SVMNT transporters ${ }^{47}$. This possibility is discussed in more detail below and in Fig. 6 .

The involvement of residue 86 in QN resistance has been controversial $^{48,49}$. Our results indicate that residue 86 of PfMDR1 plays a minor, background-specific role in QN susceptibility, with N86Y producing a slight increase in QN resistance only in NF10 parasites (that carry CVIET PfCRT). Mutant PfCRT is known to contribute to QN resistance, although the full repertoire of parasite determinants remains to be identified $48,50,51$.

Whereas the N86Y mutation affected the susceptibility of parasites to CQ and md-ADQ (and to a lesser extent QN), N86 and N86Y clones in the NF10 background showed no discernible difference in their accumulation of radiolabelled CQ, ADQ or QN (Supplementary Fig. 3). This suggests that if PfMDR1-mediated drug transport was occurring in parasites expressing a given PfMDR1 variant, the impact on drug accumulation was modest and beyond the limits of assay detection. Our data recall a prior analysis of the progeny of the GB $4 \times 7$ G8 genetic cross, which found that $p f m d r 1$ alleles could affect parasite drug susceptibility (as quantified using $\mathrm{IC}_{50}$ assays) without significantly altering
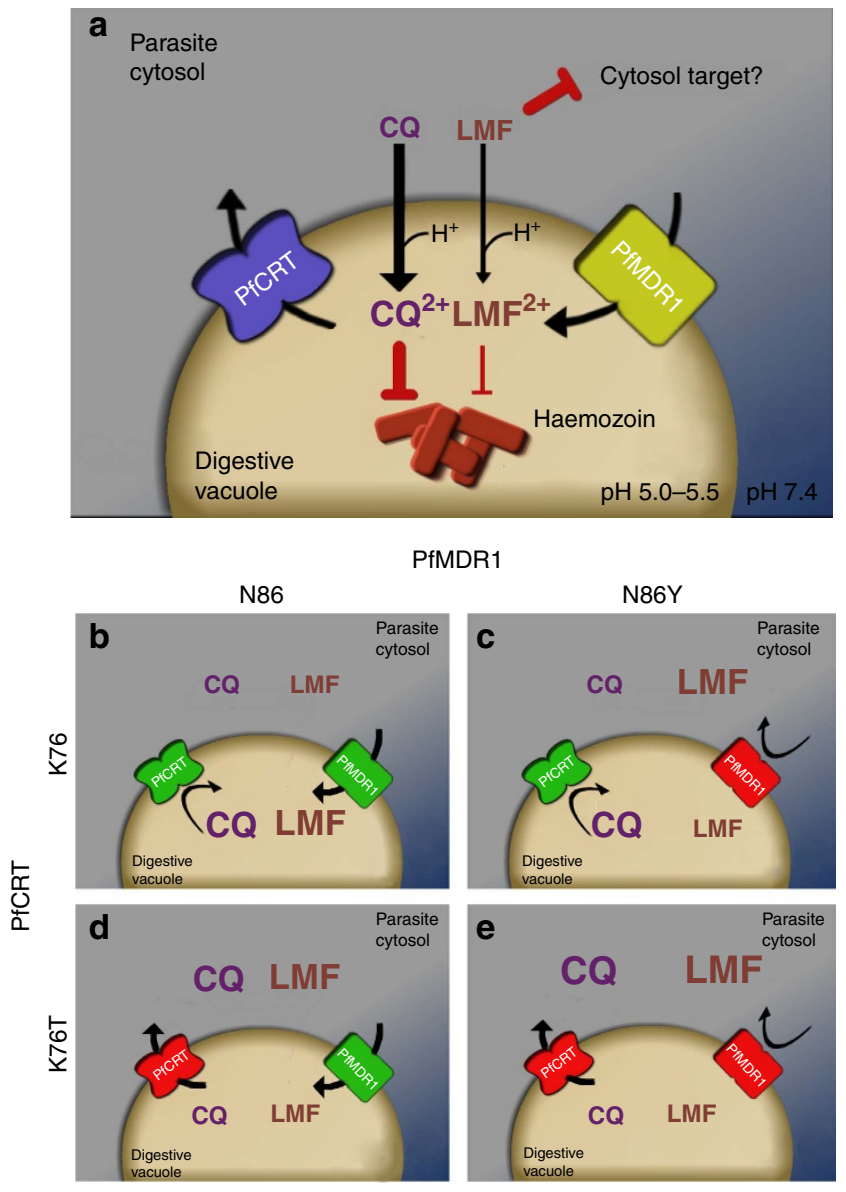

Figure 6 | Proposed roles for PfMDR1 and PfCRT in the P. falciparum response to CQ and LMF. (a) CQ (a weak base) and the hydrophobic drug LMF are thought to enter the DV via simple diffusion across the membrane. Within the DV, CQ is thought to trigger parasite killing by binding to reactive haem moieties and preventing their biomineralization into chemically inert haemozoin crystals ${ }^{67}$. LMF also shows moderate binding to haem ${ }^{2}$; however, its main site of action is suspected to be outside of the DV. It is thought that PfCRT facilitates the efflux of drugs from the DV, and that PfMDR1 transports drugs into the $D^{3,68}$. (b-e) Point mutations in these transporters are known to differentially impact parasite susceptibility to CQ and LMF. The N86Y mutation is thought to ablate PFMDR1-mediated CQ transport activity22, but the resulting reduction in the rate of $C Q$ influx is insufficient to confer $\mathrm{CQ}$ resistance in parasites that carry wild-type PfCRT (designated as K76) ${ }^{47}$ $(\mathbf{b}, \mathbf{c})$. CQ resistance-conferring isoforms of PfCRT (designated K76T) mediate the efflux of CQ from the DV ${ }^{68-70}$. The reduced rate of CQ influx in PfMDR1 N86Y parasites may further decrease CQ concentrations in the DV and thus augment CQ resistance. However, this effect is only evident in parasites expressing a high-capacity transporter of CQ (for example, GB4 PfCRT) ${ }^{47}$ This is perhaps because the loss of CQ influx via PfMDR1 causes a substantial decrease in the time taken to reach sub-lethal levels of CQ in the DV only when the drug is being extruded from the DV via K76T PfCRT at a relatively high rate. (e) LMF might also be a substrate of PfMDR1 N86 (b,d) and the introduction of N86Y $(\mathbf{c}, \mathbf{e})$ might likewise reduce the capacity of PfMDR1 to import LMF into the DV. In this scenario, the heightened susceptibility of CQresistant N86Y PfMDR1 parasites to LMF could be due to the increased accumulation of LMF in the cytosol (where its main antimalarial target may be located). The relative sizes of the CQ and LMF text in these panels reflect the predicted distribution of the drugs between the DV and cytosol.

levels of drug accumulation ${ }^{51}$. These findings suggest that polymorphisms within PfMDR1 and PfCRT can contribute to resistance phenotypes through mechanisms based not only on 
regulating drug accumulation inside the DV. One hypothesis, for which supporting evidence exists ${ }^{52-54}$, is that these antimalarials might bind these transporters directly and inhibit their physiological function. This mode of action would be in addition to these drugs interfering with reactive haem detoxification through interactions with haem ${ }^{2}$.

Our studies also revealed only a minimal impact of the Y184F mutation on antimalarial drug susceptibilities. In the NF10 background harbouring the predominant Asian/African $p f c r t$ allele, there was a decreased susceptibility to PPQ in Y184F parasites, which was observed in the presence of the N86 residue. Structural models for PfMDR1 place residue 184 in the third transmembrane domain and it has been hypothesized that the Y184F mutation might affect the kinetics of PfMDR1-mediated drug transport ${ }^{22,55}$. Transport studies in Xenopus oocytes have also provided evidence of an effect of both the Y184F and N86Y mutations on the ability of PfMDR1 to transport different drugs ${ }^{22,55}$. Studies with proteoliposomes also provide evidence that PfMDR1 or PfCRT isoforms can differentially bind quinoline drugs ${ }^{52}$.

Previous studies have used traditional transfection-based approaches to explore the effects of the C-terminal mutations S1034C, N1042D and D1246Y on parasite drug responses ${ }^{23,24}$. These C-terminal PfMDR1 mutations, which are more frequent in low-transmission areas in South America than in Asia or Africa (as obtained from www.malariagen.net/pf3k), were found to influence parasite susceptibility to MFQ, QN and ART. These mutations also had an impact on the parasite's response to CQ in some genetic backgrounds ${ }^{23,24}$. Studies with isogenic transfectant lines have also revealed that an increase in $p f m d r 1$ copy number, which is very rare in Africa but which occurs relatively frequently in parts of Southeast Asia, reduces parasite susceptibility to MFQ, LMF, QN and $\mathrm{ART}^{56}$. Our study now elucidates the roles of the other two major PfMDR1 polymorphisms, N86Y and Y184F, which are the most prevalent variations in Africa. Of note, recent studies from Uganda have observed selection for PfMDR1 wildtype N86 and D1246 in patients treated with ATM + LMF, contrasting with selection of the mutations N86Y and D1246Y in patients receiving $\mathrm{AS}+\mathrm{ADQ}^{57}$. Our evidence that $\mathrm{N} 86 \mathrm{Y}$ increases parasite susceptibility to MFQ and LMF also helps explain why this mutation is very rarely observed in Thai parasites that have been under selection with these drugs, and that often carry multicopy $p f m d r 1$, which has the opposite effect of reducing susceptibility (Fig. 4 and Supplementary Table 1). Our efficient gene-editing method now enables the examination of additional PfMDR1 polymorphisms that have recently been identified in field isolates, presumably as a result of the change in the past decade from CQ to ACTs.

Our study lends further support to the notion that the parasite genetic background is an important factor in determining the impact of PfMDR1 polymorphisms on parasite drug response. Studies with progeny of the GB4 $\times 7 \mathrm{G} 8$ cross have implicated $\mathrm{PfCRT}$ as a major driver of these divergent responses ${ }^{19}$. Evidence from heterologous expression studies in Xenopus oocytes suggest that GB4 PfCRT is a relatively high-capacity transporter of $\mathrm{CQ}$, whereas the 7G8 isoform has a much lower capacity (but somewhat higher affinity) for CQ transport ${ }^{47}$. GB4 PfCRT might therefore be effective in decreasing high concentrations of $\mathrm{CQ}$ within the DV to sub-lethal levels. In this scenario the simultaneous action of an additional mechanism for reducing CQ accumulation-for example, mutations in PfMDR1 that ablate its ability to import CQ into the DV-could translate into further increases in the parasite's resistance to CQ that are attributable to the PfMDR1 variant. By contrast, 7G8 PfCRT might act more slowly to reduce CQ concentrations to sub-lethal levels. During that time the reduced CQ transport activity of the PfMDR1 variant might be mostly or completely masked, because, even with the reduced rate of CQ influx into the DV, the vacuolar concentration of CQ would be such that parasite growth and/or viability is still significantly reduced. This is but one possible mechanistic explanation for our findings (Fig. 6) and further studies are required to delineate the mechanisms by which different variants of PfCRT and PfMDR1 combine to affect drug susceptibility.

The recent availability of thousands of $P$. falciparum genomes now makes it possible to also examine regional associations between PfMDR1 and PfCRT haplotypes (Table 1 and Supplementary Table 1). In Africa, nearly $70 \%$ of the recently sequenced isolates carried the CQ-sensitive PfCRT CVMNK (residue 72-76) haplotype, with the remainder carrying the CQ-resistant CVIET haplotype. This recent resurgence of CQ-sensitive isolates in Africa that harbour wild-type $p f c r t$ emphasizes the value of expanding $p f m d r 1$ gene-editing studies to those parasites. In Africa, the most abundant PfMDR1 N-terminal haplotypes were NF and NY. In Asia, the PfCRT haplotypes were almost all CVIET (80\%) with the remainder being the CVIDT variant that was observed to have a minimal fitness cost compared with CVIET parasites ${ }^{58}$. Asian parasites were almost all NF or NY, with the latter associating the most with the CVIDT haplotype.

Our findings also provide new insights into the ways in which drug pressures have shaped different parasite populations. In Thailand, the reliance on $\mathrm{AS}+\mathrm{MFQ}^{59}$ might explain why fewer than $1 \%$ of Thai isolates harbour the PfMDR1 N86Y mutation (Supplementary Table 1), which based on our data increases parasite susceptibility to both MFQ and DHA, and which therefore would be selected against. Indeed, MFQ has been used widely throughout Southeast Asia, which could explain the overall rarity of the N86Y mutation in this region. Bangladesh is an exception in that it has a significant proportion $(22 \%)$ of N86Y isolates. This prevalence may reflect the fact that the national malaria control programme in Bangladesh administers CQ and QN in addition to ATM $+\mathrm{LMF}^{60}$. We find that PfMDR1 N86Y decreases parasite susceptibility to both CQ and QN, suggesting its active selection in this country. The N86Y mutation also decreases parasite susceptibility to md-ADQ, which could explain why this mutation is commonly observed in West Africa where $\mathrm{AS}+\mathrm{ADQ}$ is frequently used. Malawi, on the other hand, relies on ATM + LMF as its first-line ACT, and in this east African country $<2 \%$ of parasite isolates have the LMF-sensitizing N86Y mutation. Given the rapid changes in antimalarial drug use in Africa over the past 15 years, it will be of interest to assess changes over time in pfmdr1 positions 86, 184 and 1246. The currently available genome data sets, however, do not currently permit this chronological analysis. Regarding PfMDR1 184, Laos is the only Southeast Asian country shown in Fig. 2 that employs $\mathrm{ATM}+\mathrm{LMF}$ as its sole ACT, with the other countries having a treatment policy that includes the use of $\mathrm{DHA}+\mathrm{PPQ}^{61}$. In light of our finding that the NF haplotype is associated with slightly decreased susceptibility to PPQ in parasites harbouring the Southeast Asian/African CVIET variant of PfCRT, we propose that the difference in treatment policies explains the rarity of the Y184F mutation in Laos ( $<4 \%$ of isolates) and its elevated frequency ( $>20 \%$ of isolates) in the other Southeast Asian countries that more commonly use DHA + PPQ. Based on our combined phenotypic and mapping data, we propose that an optimal policy for ACT usage would entail the use of LMF in areas with a high proportion of N86Y, ADQ in areas where N86 is prevalent and PPQ in areas with a high frequency of NY haplotype. The competing selective forces of LMF and ADQ, both on PfMDR1 and PfCRT ${ }^{15,62}$, also support scenarios of dual implementation of ATM + LMF and AS + ADQ, to suppress the emergence of high-grade multidrug resistance. 


\section{Methods}

Analysis of the Pf3k P. falciparum genome data set. Genome analysis used data generated by the Pf3k project (www.malariagen.net/pf3k ${ }^{8,29}$. Assembly files were downloaded from $\mathrm{ftp} / / /$ ngs.sanger.ac.uk/production/pf3k/release_3/, a publically accessible database. SNPs were manually extracted using the annotated Pf3D7 genome version 9.2 (the same used to assemble the field isolate genomes) and a custom Perl script used to call each base, its sample depth and allelic balance. Indels were not considered. The output was inserted as a table into a MySQL database that contained Pf3k metadata (including isolate origins and general mapping quality values). Using bash shell-MySQL connectors, we retrieved PfMDR1 (PF3D7_0523000) haplotype data from the database in an ordered list (separated by geographic region) on a per-genome basis. Haplotypes for amino acids 86 and 184 were counted and visualized in Excel. Copy number variations (CNVs) were assessed using the programme BICSeq ${ }^{63}$, incorporated in a shell script to permit parallel processing. Analysis was performed iteratively against several reference genomes to find the closest match for each field isolate. These included the West African laboratory strain 3D7, the Cambodian isolate PH0164-C and the Senegalese isolate SenT087.09. PH0164-C has a Southeast Asian PfCRT haplotype (eight mutations), no $p f m d r 1$ or $k 13$ mutations and no known $p f m d r 1$ CNVs. SenT087.09 has no CNVs, is wild-type for $p f c r t$ and $p f m d r 1$, and carries a triple mutant pfdhfr (encoding N51I, C59L and S108N) that is common in Africa and confers pyrimethamine resistance. Default parameters were set to a bin size of 300 nucleotides, a window size of 600 nucleotides and a smoothing factor of 12 . To plot the distribution of CNVs, values were combined per country and separated by non-CNV-harbouring isolates and CNV-harbouring isolates of a certain haplotype. The geographical map was downloaded using free spatial data from DIVA-GIS (http://diva-gis.org) and imported into QGIS 2.10 (http://qgis.org/en/site) to be merged with the geo-referenced data. This map was overlaid with the previously calculated haplotypes as pie charts.

Plasmid construction. CompoZr Custom ZFNs were purchased from Sigma-Aldrich (St. Louis, Missouri, USA) to induce a double-strand break in the $5^{\prime}$-end of the pfmdr1 coding sequence. Two ZFNs (left and right), binding to adjacent sequences on opposite strands of the DNA helix, are required to induce the double-strand break. These were supplied on separate plasmids. The ZFN pair was designed to bind to the sequence $5^{\prime}$-GGTAACCTCAGTatcaaAGAAGAGG TTGAAAAAGA-3' (the DNA cut site is shown in lower case letters and is situated 42 bp downstream of the $p f m d r 1$ start codon). The plasmid encoding the right ZFN also encoded the $2 \mathrm{~A}$ 'ribosome skip' peptide, which enables polycistronic expression of $2 \mathrm{~A}$-linked genes ${ }^{26}$. The two plasmids encoding the ZFNs were digested with BglII and XhoI, and combined to yield an intermediate plasmid with 2A-linked ZFN sequences. The ZFN fusion was then digested with NheI and XhoI, and subcloned downstream of a calmodulin (PF14_0323) promoter and upstream of the hsp86 (PF07_0030) 3'-untranslated region in a pDC2-based vector with the hdhfr selectable marker ${ }^{26}$, yielding the plasmid pZFN ${ }^{p f m d r l}$-hdhfr.

A 2.4-kb donor sequence, encompassing $555 \mathrm{bp}$ of the $p f m d r 15^{\prime}$-untranslated region and 1,805 bp of the pfmdr1 coding sequence, was amplified from NF54 genomic DNA with the primers $\mathrm{p} 1+\mathrm{p} 2$ (Supplementary Table 8) and inserted into the BstAPI and AatII sites of pZFN ${ }^{p f m d r 1}$-hdhfr to yield the editing plasmid pmdr1. To prevent edited genomes from being further cleaved, three silent mutations were engineered into the donor sequence at the ZFN-binding site via site-directed mutagenesis (QuikChange Multi SDM Kit; Agilent Technologies, Santa Clara, California, USA) using the primers $\mathrm{p} 9+\mathrm{p} 10$. The donor template, containing the three silent mutations, was further mutated as required to introduce point mutations at $p f m d r 1$ codons 86 and 184 (using the primer pairs $\mathrm{p} 11+\mathrm{p} 12$ or p13 + p14), yielding four transfection plasmids (Fig. 3).

Parasite culture and transfection. P. falciparum NF10 and KC5 clones (kindly provided by Dr Thomas E. Wellems, NIH) were maintained at $\sim 3 \%$ haematocrit with human $\mathrm{O}^{+}$RBCs (Interstate Blood Bank, Memphis, TN, USA) in RPMI-1640 supplemented with $2 \mathrm{gl}^{-1}$ sodium bicarbonate, $2 \mathrm{mM}$ L-glutamine, $25 \mathrm{mM}$ HEPES, $10 \mu \mathrm{g} \mathrm{ml}^{-1}$ gentamycin, $50 \mu \mathrm{M}$ hypoxanthine and $0.5 \%(\mathrm{w} / \mathrm{v})$ AlbuMAXII (Invitrogen, Waltham, Massachusetts, USA). They were maintained at $37^{\circ} \mathrm{C}$ in an airtight environment flushed with $5 \% \mathrm{O}_{2} / 5 \% \mathrm{CO}_{2} / 90 \% \mathrm{~N}_{2}$. Ring-stage cultures at $5-8 \%$ parasitaemia were electroporated with $\sim 70 \mu \mathrm{g}$ of purified plasmid DNA eluted in $1 \times$ Cytomix, as described ${ }^{26}$

Four different plasmids expressing the PfMDR1 86/184 residues (YF, YY, NF or $\mathrm{NY}$ ) were used to electroporate NF10 and KC5 cultures. The plasmids were designed to modify pfmdr1 codons 86 and 184, and to integrate three silent mutations at the ZFN-binding site. To enable transient ZFN expression and consequent homology repair, WR99210 (2.5 nM; Jacobus Pharmaceuticals, Plainsboro, New Jersey, USA) was applied to transfected cultures $24 \mathrm{~h}$ postelectroporation and maintained for 4-6 days. We cloned edited parasites by limiting dilution in 96-well plates.

DNA analysis. Clones from transfected NF10 and KC5 parasites were checked for ZFN-mediated editing by PCR amplification from genomic DNA using the KAPA Blood PCR Kit B (KAPA Biosystems, Wilmington, Massachusetts, USA) and the primers p3 (located upstream of the pfmdr1 start codon and not in the plasmid 'donor sequence') and p4 (Supplementary Table 8). The silent mutations introduced into the donor sequence create an SmlI restriction site, which was used to screen PCR products. Amplified fragments containing the silent mutations were sequenced to confirm editing at codons 86 and 184. Successfully edited clones were expanded for further analysis. $p f m d r 1$ copy number analysis was performed using a published Taqman-based quantification assay ${ }^{35}$. 3D7 and FCB were used as control parasites, as they harbour 1 and 2 copies of $p f m d r 1$, respectively (Supplementary Table 2).

In vitro antimalarial drug assays. Drug inhibitory assays were performed using a flow cytometry-based method described previously ${ }^{64}$, with some modifications. Assays were performed in flat-bottom 96-well plates, with each well containing $200 \mu \mathrm{l}$ of cell suspension in culture medium, with or without the test compound as appropriate. The starting haematocrit was $1 \%$ and the starting parasitaemia (consisting of predominantly ring-stage parasites) was $0.1-0.2 \%$. After $72 \mathrm{~h}$ incubation at $37^{\circ} \mathrm{C}$, samples were analysed by flow cytometry. Cells were stained with $1.6 \mu \mathrm{M}$ Mito Tracker Deep Red and $2 \times$ SYBR Green (Invitrogen) in $1 \times \mathrm{PBS}^{64}$. In vitro $\mathrm{IC}_{50}$ and $\mathrm{IC}_{90}$ values were calculated using nonlinear regression analysis. In vitro Ring-stage Survival Assay $\left(\mathrm{RSA}_{0-3 \mathrm{~h}}\right)$ and Trophozoite-stage Survival Assay (TSA TS-21h $_{10}$ ) were carried out with $700 \mathrm{nM}$ DHA as previously described $^{7,34}$. In summary, NF10 recombinant and parental lines were sorbitol and Percoll synchronized and tested as $0-3 \mathrm{~h}$ post-invasion ring stages and $18-21 \mathrm{~h}$ post-invasion trophozoites. Parasites were adjusted to $1 \%$ parasitaemia and exposed to $700 \mathrm{nM}$ DHA for $6 \mathrm{~h}$. After this time, drug was removed by washing and parasites were cultured a further $66 \mathrm{~h}$. Parasite viability was assessed by flow cytometry as stated above. RBCs $(20,000$ to 35,000$)$ were counted in two separate wells per experiment, which was repeated on three separate occasions ${ }^{7}$. Percent survival was calculated as the parasitaemia in the drug-treated sample divided by the parasitaemia in the untreated sample $\times 100$ (Supplementary Fig. 2).

Drug accumulation assays. Assays were performed on mature, intact trophozoite-infected erythrocytes suspended in bicarbonate-free RPMI 1640 medium (supplemented with $25 \mathrm{mM}$ HEPES, $10 \mathrm{mM}$ glucose and $0.2 \mathrm{mM}$ hypoxanthine, and adjusted to $\mathrm{pH} 7.4$ ) at a parasitaemia of $3.7-9.1 \%$ and a haematocrit of $2 \%$. The final concentrations of $\left[{ }^{3} \mathrm{H}\right] \mathrm{CQ}\left(20 \mathrm{Ci} \mathrm{mmol}^{-1}\right),\left[{ }^{3} \mathrm{H}\right]$ QN $\left(80 \mathrm{Ci} \mathrm{mmol}^{-1}\right)$ and $\left[{ }^{3} \mathrm{H}\right]$ ADQ $\left(15 \mathrm{Ci} \mathrm{mmol}^{-1}\right)$ were 20,20 and $40 \mathrm{nM}$, respectively. Labelled drugs were purchased from American Radiolabeled Chemicals. Assays were performed according to a protocol described previously ${ }^{65}$ with one modification: the incorporation of radiolabelled drug into the acidinsoluble pellets was also measured ${ }^{66}$ and values included in determinations of the amount of drug taken up by the cells. Accumulation studies were also attempted with radiolabelled LMF; however, a high level of variability was observed. This may have resulted from the poor solubility of LMF in one of the solutions used in the experiment and/or from the diffusion of LMF into the oil layer that was used to terminate drug accumulation (the cells are separated from the radiolabelled drug solution by centrifugation through oil).

Statistical tests. All statistical analyses used non-parametric two-tailed Mann-Whitney $U$-tests (normal distribution not assumed; performed with GraphPad Prism Software).

\section{References}

1. WHO. Malaria World Report. http://www.who.int/malaria/publications/worldmalaria-report-2015/en (2015).

2. Combrinck, J. M. et al. Insights into the role of heme in the mechanism of action of antimalarials. ACS Chem. Biol. 8, 133-137 (2013).

3. Rohrbach, P. et al. Genetic linkage of $p f m d r 1$ with food vacuolar solute import in Plasmodium falciparum. EMBO J. 25, 3000-3011 (2006).

4. Klonis, N., Creek, D. J. \& Tilley, L. Iron and heme metabolism in Plasmodium falciparum and the mechanism of action of artemisinins. Curr. Opin. Microbiol. 16, $722-727$ (2013).

5. Ashley, E. A. et al. Spread of artemisinin resistance in Plasmodium falciparum malaria. N. Engl. J. Med. 371, 411-423 (2014).

6. Ariey, F. et al. A molecular marker of artemisinin-resistant Plasmodium falciparum malaria. Nature 505, 50-55 (2014).

7. Straimer, J. et al. K13-propeller mutations confer artemisinin resistance in Plasmodium falciparum clinical isolates. Science 347, 428-431 (2015).

8. Miotto, O. et al. Genetic architecture of artemisinin-resistant Plasmodium falciparum. Nat. Genet. 47, 226-234 (2015).

9. Saunders, D. L. et al. Dihydroartemisinin-piperaquine failure in Cambodia. N. Engl. J. Med. 371, 484-485 (2014).

10. Leang, R. et al. Evidence of Plasmodium falciparum malaria multidrug resistance to artemisinin and piperaquine in Western Cambodia: dihydroartemisinin-piperaquine open-label multicenter clinical assessment. Antimicrob. Agents Chemother. 59, 4719-4726 (2015).

11. Amaratunga, C. et al. Dihydroartemisinin-piperaquine resistance in Plasmodium falciparum malaria in Cambodia: a multisite prospective cohort study. Lancet Infect. Dis. 16, 357-365 (2016). 
12. Wells, T. N., Hooft van Huijsduijnen, R. \& Van Voorhis, W. C. Malaria medicines: a glass half full? Nat. Rev. Drug Discov. 14, 424-442 (2015).

13. Malmberg, M. et al. Plasmodium falciparum drug resistance phenotype as assessed by patient antimalarial drug levels and its association with pfmdr1 polymorphisms. J. Infect. Dis. 207, 842-847 (2013).

14. Conrad, M. D. et al. Comparative impacts over 5 years of artemisinin-based combination therapies on Plasmodium falciparum polymorphisms that modulate drug sensitivity in Ugandan children. J. Infect. Dis. 210, 344-353 (2014).

15. Venkatesan, M. et al. Polymorphisms in Plasmodium falciparum chloroquine resistance transporter and multidrug resistance 1 genes: parasite risk factors that affect treatment outcomes for $P$. falciparum malaria after artemether-lumefantrine and artesunate-amodiaquine. Am. J. Trop. Med. Hyg. 91, 833-843 (2014).

16. de Koning-Ward, T. F., Gilson, P. R. \& Crabb, B. S. Advances in molecular genetic systems in malaria. Nat. Rev. Microbiol. 13, 373-387 (2015).

17. Valderramos, S. G. \& Fidock, D. A. Transporters involved in resistance to antimalarial drugs. Trends Pharmacol. Sci. 27, 594-601 (2006).

18. Happi, C. T. et al. Linkage disequilibrium between two distinct loci in chromosomes 5 and 7 of Plasmodium falciparum and in vivo chloroquine resistance in Southwest Nigeria. Parasitol. Res. 100, 141-148 (2006).

19. Sa, J. M. et al. Geographic patterns of Plasmodium falciparum drug resistance distinguished by differential responses to amodiaquine and chloroquine. Proc. Natl Acad. Sci. USA 106, 18883-18889 (2009).

20. Fidock, D. A. et al. Mutations in the P. falciparum digestive vacuole transmembrane protein PfCRT and evidence for their role in chloroquine resistance. Mol. Cell 6, 861-871 (2000).

21. Lekostaj, J. K., Amoah, L. E. \& Roepe, P. D. A single S1034C mutation confers altered drug sensitivity to PfMDR1 ATPase activity that is characteristic of the 7G8 isoform. Mol. Biochem. Parasitol. 157, 107-111 (2008).

22. Sanchez, C. P., Rotmann, A., Stein, W. D. \& Lanzer, M. Polymorphisms within PfMDR1 alter the substrate specificity for anti-malarial drugs in Plasmodium falciparum. Mol. Microbiol. 70, 786-798 (2008).

23. Reed, M. B., Saliba, K. J., Caruana, S. R., Kirk, K. \& Cowman, A. F. Pgh1 modulates sensitivity and resistance to multiple antimalarials in Plasmodium falciparum. Nature 403, 906-909 (2000).

24. Sidhu, A. B., Valderramos, S. G. \& Fidock, D. A. $p f m d r 1$ mutations contribute to quinine resistance and enhance mefloquine and artemisinin sensitivity in Plasmodium falciparum. Mol. Microbiol. 57, 913-926 (2005).

25. Lee, A. H., Symington, L. S. \& Fidock, D. A. DNA repair mechanisms and their biological roles in the malaria parasite Plasmodium falciparum. Microbiol. Mol. Biol. Rev. 78, 469-486 (2014).

26. Straimer, J. et al. Site-specific genome editing in Plasmodium falciparum using engineered zinc-finger nucleases. Nat. Methods 9, 993-998 (2012).

27. McNamara, C. W. et al. Targeting Plasmodium PI(4)K to eliminate malaria. Nature 504, 248-253 (2013).

28. Pelleau, S. et al. Adaptive evolution of malaria parasites in French Guiana: reversal of chloroquine resistance by acquisition of a mutation in pfcrt. Proc. Natl Acad. Sci. USA 112, 11672-11677 (2015).

29. MalariaGEN Plasmodium falciparum Community Project. Genomic epidemiology of artemisinin resistant malaria. Elife 5, pii: e08714 (2016).

30. Rosenthal, P. J. The interplay between drug resistance and fitness in malaria parasites. Mol. Microbiol. 89, 1025-1038 (2013).

31. Takala-Harrison, S. \& Laufer, M. K. Antimalarial drug resistance in Africa: key lessons for the future. Ann. N. Y. Acad. Sci. 1342, 62-67 (2015).

32. Hayton, K. et al. Erythrocyte binding protein PfRH5 polymorphisms determine species-specific pathways of Plasmodium falciparum invasion. Cell Host Microbe 4, 40-51 (2008).

33. Birth, D., Kao, W. C. \& Hunte, C. Structural analysis of atovaquone-inhibited cytochrome bcl complex reveals the molecular basis of antimalarial drug action. Nat. Commun. 5, 4029 (2014).

34. Witkowski, B. et al. Novel phenotypic assays for the detection of artemisininresistant Plasmodium falciparum malaria in Cambodia: in-vitro and ex-vivo drug-response studies. Lancet Infect. Dis. 13, 1043-1049 (2013).

35. Price, R. N. et al. Mefloquine resistance in Plasmodium falciparum and increased pfmdr1 gene copy number. Lancet 364, 438-447 (2004).

36. Ekland, E. H. \& Fidock, D. A. In vitro evaluations of antimalarial drugs and their relevance to clinical outcomes. Int. J. Parasitol. 38, 743-747 (2008).

37. Price, R. N. et al. The pfmdr1 gene is associated with a multidrug-resistant phenotype in Plasmodium falciparum from the western border of Thailand. Antimicrob. Agents Chemother. 43, 2943-2949 (1999).

38. Imwong, M. et al. Exploring the contribution of candidate genes to artemisinin resistance in Plasmodium falciparum. Antimicrob. Agents Chemother. 54, 2886-2892 (2010).

39. Veiga, M. I. et al. Novel polymorphisms in Plasmodium falciparum ABC transporter genes are associated with major ACT antimalarial drug resistance. PLOS ONE 6, e20212 (2011).
40. Pickard, A. L. et al. Resistance to antimalarials in Southeast Asia and genetic polymorphisms in pfmdrl. Antimicrob. Agents Chemother. 47, 2418-2423 (2003).

41. Tumwebaze, P. et al. Impact of antimalarial treatment and chemoprevention on the drug sensitivity of malaria parasites isolated from ugandan children. Antimicrob. Agents Chemother. 59, 3018-3030 (2015).

42. Sisowath, C. et al. In vivo selection of Plasmodium falciparum pfmdr1 86N coding alleles by artemether-lumefantrine (Coartem). J. Infect. Dis. 191, 1014-1017 (2005).

43. Sigala, P. A. \& Goldberg, D. E. The peculiarities and paradoxes of Plasmodium heme metabolism. Annu. Rev. Microbiol. 68, 259-278 (2014).

44. $\mathrm{Mu}$, J. et al. Multiple transporters associated with malaria parasite responses to chloroquine and quinine. Mol. Microbiol. 49, 977-989 (2003).

45. Holmgren, G. et al. Amodiaquine resistant Plasmodium falciparum malaria in vivo is associated with selection of pfcrt 76T and pfmdr1 86Y. Infect. Genet Evol. 6, 309-314 (2006).

46. Zongo, I. et al. Artemether-lumefantrine versus amodiaquine plus sulfadoxine-pyrimethamine for uncomplicated falciparum malaria in Burkina Faso: a randomised non-inferiority trial. Lancet 369, 491-498 (2007).

47. Summers, R. L. et al. Diverse mutational pathways converge on saturable chloroquine transport via the malaria parasite's chloroquine resistance transporter. Proc. Natl Acad. Sci. USA 111, E1759-E1767 (2014).

48. Ferdig, M. T. et al. Dissecting the loci of low-level quinine resistance in malaria parasites. Mol. Microbiol. 52, 985-997 (2004).

49. Wurtz, N. et al. Role of Pfmdr1 in in vitro Plasmodium falciparum susceptibility to chloroquine, quinine, monodesethylamodiaquine, mefloquine, lumefantrine, and dihydroartemisinin. Antimicrob. Agents Chemother. 58, 7032-7040 (2014).

50. Sidhu, A. B., Verdier-Pinard, D. \& Fidock, D. A. Chloroquine resistance in Plasmodium falciparum malaria parasites conferred by pfcrt mutations. Science 298, 210-213 (2002).

51. Sanchez, C. P., Mayer, S., Nurhasanah, A., Stein, W. D. \& Lanzer, M. Genetic linkage analyses redefine the roles of PfCRT and PfMDR1 in drug accumulation and susceptibility in Plasmodium falciparum. Mol. Microbiol. 82, 865-878 (2011).

52. Pleeter, P., Lekostaj, J. K. \& Roepe, P. D. Purified Plasmodium falciparum multi-drug resistance protein (PfMDR1) binds a high affinity chloroquine analogue. Mol. Biochem. Parasitol. 173, 158-161 (2010).

53. Roepe, P. D. PfCRT-mediated drug transport in malarial parasites. Biochemistry 50, 163-171 (2011)

54. Summers, R. L., Nash, M. N. \& Martin, R. E. Know your enemy: understanding the role of PfCRT in drug resistance could lead to new antimalarial tactics. Cell. Mol. Life Sci. 69, 1967-1995 (2012).

55. Ferreira, P. E. et al. PfMDR1: mechanisms of transport modulation by functional polymorphisms. PLoS ONE 6, e23875 (2011).

56. Sidhu, A. B. et al. Decreasing pfmdr1 copy number in Plasmodium falciparum malaria heightens susceptibility to mefloquine, lumefantrine, halofantrine, quinine, and artemisinin. J. Infect. Dis. 194, 528-535 (2006).

57. Yeka, A. et al. Artesunate/amodiaquine versus artemether/lumefantrine for the treatment of uncomplicated malaria in Uganda: a randomized trial. J. Infect. Dis. 213, 1134-1142 (2016).

58. Petersen, I. et al. Balancing drug resistance and growth rates via compensatory mutations in the Plasmodium falciparum chloroquine resistance transporter. Mol. Microbiol. 97, 381-395 (2015).

59. Carrara, V. I. et al. Changes in the treatment responses to artesunatemefloquine on the northwestern border of Thailand during 13 years of continuous deployment. PLoS ONE 4, e4551 (2009).

60. Haque, U. et al. Malaria burden and control in Bangladesh and prospects for elimination: an epidemiological and economic assessment. Lancet Glob. Health 2, e98-e105 (2014).

61. O’Brien, C., Henrich, P. P., Passi, N. \& Fidock, D. A. Recent clinical and molecular insights into emerging artemisinin resistance in Plasmodium falciparum. Curr. Opin. Infect. Dis. 24, 570-577 (2011).

62. Ecker, A., Lehane, A. M. \& Fidock, D. A. Molecular markers of Plasmodium resistance to antimalarials. In: Treatment and prevention of malaria antimalarial drug chemistry, action and use (eds Staines, H. M. \& Krishna, S.) (Springer, 2012).

63. Xi, R. et al. Copy number variation detection in whole-genome sequencing data using the Bayesian information criterion. Proc. Natl Acad. Sci. USA 108, E1128-E1136 (2011).

64. Ekland, E. H., Schneider, J. \& Fidock, D. A. Identifying apicoplast-targeting antimalarials using high-throughput compatible approaches. FASEB J. 25, 3583-3593 (2011).

65. Lehane, A. M., van Schalkwyk, D. A., Valderramos, S. G., Fidock, D. A. \& Kirk, K. Differential drug efflux or accumulation does not explain variation in the chloroquine response of Plasmodium falciparum strains expressing the 
same isoform of mutant PfCRT. Antimicrob. Agents Chemother. 55, 2310-2318 (2011).

66. Martin, R. E. \& Kirk, K. Transport of the essential nutrient isoleucine in human erythrocytes infected with the malaria parasite Plasmodium falciparum. Blood 109, 2217-2224 (2007).

67. Ecker, A., Lehane, A. M., Clain, J. \& Fidock, D. A. PfCRT and its role in antimalarial drug resistance. Trends Parasitol. 28, 504-514 (2012).

68. Martin, R. E. et al. Chloroquine transport via the malaria parasite's chloroquine resistance transporter. Science 325, 1680-1682 (2009).

69. Bellanca, S. et al. Multiple drugs compete for transport via the Plasmodium falciparum chloroquine resistance transporter at distinct but interdependent sites. J. Biol. Chem. 289, 36336-36351 (2014).

70. Callaghan, P. S., Hassett, M. R. \& Roepe, P. D. Functional comparison of 45 naturally occurring isoforms of the Plasmodium falciparum chloroquine resistance transporter (PfCRT). Biochemistry 54, 5083-5094 (2015).

\section{Acknowledgements}

This work was funded in part by the National Institutes of Health (R01 AI50234, AI124678 and AI109023) and a Burroughs Wellcome Fund Investigator in Pathogenesis of Infectious Diseases award to D.A.F. This research also received funding from the Portuguese Fundação para a Ciência e Tecnologia (FCT), cofunded by Programa Operacional Regional do Norte (ON.2-O Novo Norte); from the Quadro de Referência Estratégico Nacional (QREN) through the Fundo Europeu de Desenvolvimento Regional (FEDER) and from the Projeto Estratégico - LA 26 - 2013-2014 (PEst-C/SAU/LA0026/ 2013). M.I.V. is the recipient of a postdoctoral fellowship from FCT/Ministerio da Ciência e Ensino Superior, Portugal-MCES (SFRH/BPD/76614/2011). A.M.L. was supported by an Australian National Health and Medical Research Council (NHMRC)

Overseas Biomedical Fellowship (585519). R.E.M. was supported by an NHMRC RD

Wright Biomedical Fellowship (1053082). A.C.U. was supported by an Irving scholarship from Columbia University. We thank Dr Andrea Ecker for her help with plasmid design and Pedro Ferreira for his expert help with Fig. 6.

\section{Author contributions}

Conceived and designed the experiments: M.I.V., S.K.D., J.S., N.G., A.C.U., R.E.M., A.M.L. and D.A.F. Acquired the data: M.I.V., S.K.D., P.P.H., J.S., N.G., R.E.M. and A.M.L. Analysed and interpreted the data: M.I.V., S.K.D., P.P.H., J.S., N.G., R.E.M., A.M.L. and D.A.F. Wrote the paper: M.I.V., S.K.D. and D.A.F., with input from other authors. All authors approved the final manuscript.

\section{Additional information}

Supplementary Information accompanies this paper at http://www.nature.com/ naturecommunications

Competing financial interests: The authors declare no competing financial interests.

Reprints and permission information is available online at http://npg.nature.com/ reprintsandpermissions/

How to cite this article: Veiga, M. I. et al. Globally prevalent PfMDR1 mutations modulate Plasmodium falciparum susceptibility to artemisinin-based combination therapies. Nat. Commun. 7:11553 doi: 10.1038/ncomms11553 (2016).

\section{(c) (i)}

This work is licensed under a Creative Commons Attribution 4.0 International License. The images or other third party material in this article are included in the article's Creative Commons license, unless indicated otherwise in the credit line; if the material is not included under the Creative Commons license, users will need to obtain permission from the license holder to reproduce the material. To view a copy of this license, visit http://creativecommons.org/licenses/by/4.0/ 